\title{
Impact of Bone Marrow Cell Therapy on Left Ventricular Ejec- tion Fraction in Adult Patients with Heart Disease: An Applica- tion of Objective Bayesian Random-Effect Meta-Analysis
}

\author{
Siew-Pang Chan ${ }^{1 *}$, Pei-Jing Low ${ }^{2}$ \\ ${ }^{1}$ Department of Cardiology, National University of Singapore, Singapore \\ ${ }^{2}$ Cardiovascular Research Institute, National University Heart Centre Singapore, Singapore
}

"Corresponding author: Siew-Pang Chan, Department of Cardiology, National University of Singapore, Department of Mathematics \& Statistics, La Trobe University, National University Health System Tower Block Level 9 1E Kent Ridge Road, Singapore. Tel: +6590499849; Email: mdccsp@nus.edu.sg; bizv459@nus.edu.sg

Citation: Chan SP, Low PJ (2016) Impact of Bone Marrow Cell Therapy on Left Ventricular Ejection Fraction in Adult Patients with Heart Disease: An Application of Objective Bayesian Random-Effect Meta-Analysis. Cardiolog Res Cardiovasc Med 1: 102. DOI: $10.29011 / 2575-7083.000002$

Received Date: 1 June, 2016; Accepted Date: 20 June, 2016; Published Date: 4 July, 2016

\begin{abstract}
Introduction: The objective of this paper is to construct a Bayesian model for re-analyzing the results of several published metaanalyses concerning the impact of Bone Marrow Cell Therapy (BMC) on Left Ventricular Ejection Fraction (LVEF) in adult patients with heart diseases.

Method: The pooled mean difference in LVEF between BMC and the control group is the outcome of interest. With the help of the Gibbs sampler, the proposed Bayesian model combined the data from 6 cohort studies (prior) with the results collated from 54 well-cited randomized controlled trials published from 2004 to 2013 (likelihood) for generating the posterior distribution for meta-analysis.
\end{abstract}

Results: The conventional model showed that BMC could bring modest but statistically significant effect to patients with heart diseases. The pooled effect of mean difference in LVEF was $+3.12 \%$ (95\% C.I.: 2.20-4.03) in favour of BMC, according to the objective Bayesian model. Sensitivity analyses showed that the priors had little influence on the posterior results.

Discussion/Conclusion: More RCTs are needed before further conclusion can be made about the impact of BMC on LVEF. The Bayesian model has provided more insights to the problem and the nature of data selected for meta-analysis.

Keywords: Bone marrow cell therapy; Left ventricular ejection fraction; Objective bayesian meta-analysis

\section{Introduction}

Although the results of Bone Marrow Cell Therapy (BMC) are fairly inconsistent [1], several meta-analyses of pooled data from clinical trials [2-8] have shown that it could bring beneficial impact on cardiac function in adult patients with Acute Myocardial Infarction (AMI) and Ischemic Heart Disease (IHD). All but one of the above-mentioned meta-analyses was performed with the conventional statistical method involving random effects. The aim of this paper is to present a prototype Bayesian model as an alternative approach for re-analyzing the published results. This could be of interest to cardiologists as it helps to further evaluate the potential therapeutic benefits of BMC for cardiac repair with a more comprehensive approach. Moreover, Bayesian statistics has gained much attention in cardiovascular research in recent years [9] and a fruitful discussion can be found in references $[9,10]$.

Following the rationale of conventional statistical theory, the pooled effect of a meta-analysis is an unknown but fixed quantity [11] that can be estimated from combining the individual weighted study effects obtained from systematic reviews. However, a versatile model should reflect how we think and how we make decisions with available information and existing knowledge. We 


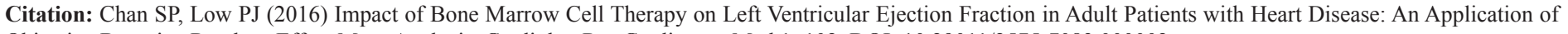
Objective Bayesian Random-Effect Meta-Analysis. Cardiolog Res Cardiovasc Med 1: 102. DOI: 10.29011/2575-7083.000002

often approach a problem and find its solution based on some preunderstanding before we "revise" our judgement with newly-collected information. As such, we often have some ideas about the pooled effect even before a meta-analysis is conducted. Building such knowledge into analysis could produce a more complete and meaningful paradigm. According to the Bayesian framework, this evidence is termed as "prior" as they are "known" before an analysis is performed. When the prior knowledge is combined with the collected data (likelihood) we revise our understanding and the resultant paradigm is called the posterior, which serves as the basis for inference and decision making.

The proposed Bayesian framework and model is discussed next. The pooled effect considered in this paper is the mean difference in Left Ventricular Ejection Fraction (LVEF) between patients treated with BMC and their controls enrolled in Randomized Controlled Trials (RCT). Reported in nearly all clinical trials of BMC therapy, LVEF reflects the overall contractile function LV [12] and has been presented as the primary endpoint of the abovementioned meta-analyses [2-8].

A common concern among scientists is that the prior and posterior advocated by Bayesian statistics represent subjective states of knowledge [13]. This paper may help to illustrate that this is far from true as a Bayesian model could be constructed with objective prior.

\section{Method}

There are three key quantities to be considered in the proposed Bayesian model, namely a) the pooled effect, b) the trialspecific effect and c) the between-trial precision. The pooled effect $(\theta)$ refers to the overall weighted mean difference in LVEF between $\mathrm{BMC}$ and the control arm, to be estimated with the $\mathrm{k}$ individual observed trial effects (y) weighted with observed within-study preci- sion $\left(\varphi_{\mathrm{i}}\right)$, which is defined as the reciprocal of the standard error of $y$. The higher the reported standard error, the lower the trial's $(\mathrm{i}=1,2, \ldots, \mathrm{k})$ precision. The two observed quantities, $\mathrm{y}$ and $\varphi_{\mathrm{i}}$, are to be presented with likelihood functions, according to statistical theory.

As reported in the abovementioned meta-analyses there was a significant degree of heterogeneity among the individual studies [2-8] owing to the fact that vastly different cell numbers were injected via different routes and at various time points in dissimilar patient populations [14]. It is expected that there are variability between the reported studies in terms of their effects and precision, thus calling for the inclusion of unobserved random effects in the proposed Bayesian model. The observed individual trial effects (y) could vary around their unobserved trial-specific effect $\left(\phi_{i}\right)$, which in turn belong to a distribution characterized by the pooled effect $(\theta)$. Both $\theta$ and the between-trial precision $(\tau)$ are random variables, and prior distributions must be considered. They quantify the analysts' pre-understanding about $\theta$ and $\tau$.

In most meta-analyses, normal distribution is appropriate for the observed effects (y). If normal distribution is not immediately appropriate, one may perform logarithmic transform on $\mathrm{y}$. The prior distributions of $\theta$ and $\tau$ are chosen within the related conjugate family such that $\theta$ belongs to a normal distribution with parameters $\mu$ (location) and $v$ (scale), while $\tau$ belongs to a gamma distribution characterized by quantities $\lambda$ (shape) and $\eta$ (scale). The concept of conjugacy ensures that the posterior distribution is mathematically tractable. Once the posterior is generated the pooled effect $\theta$ can be determined with ease.

Collecting all the terms and based on the celebrated Bayes' Theorem [15], the full posterior of the proposed model for pooling the reported effects of $\mathrm{k}$ individual trials is expressed as:

$\mathrm{p}[\theta, \phi, \tau, \varphi \mid \mathrm{y}] \prod_{\mathrm{i}=1}^{\mathrm{k}} \sqrt{\frac{\phi_{\mathrm{i}}}{2 \pi}} \exp \left[\frac{-\phi_{\mathrm{i}}}{2}\left(\mathrm{y}_{\mathrm{i}}-\varphi_{\mathrm{i}}\right)^{2}\right] \times \prod_{i=1}^{k} \sqrt{\frac{\mathrm{t}}{2 \mathrm{p}}} \exp \left[\frac{-\mathrm{t}}{2}\left(\mathrm{j}_{i}-\mathrm{q}\right)^{2}\right] \times \sqrt{\frac{\nu}{2 \pi}} \exp \left[\frac{-\mathrm{v}}{2}(\theta-\mu)^{2}\right] \times \frac{\eta^{\lambda}}{\Gamma(\lambda)} \tau^{\lambda-1} \exp [-\boldsymbol{\eta}]$

Where $y=\left(y_{1}, y_{2}, \ldots, y_{k}\right)^{\prime}$ is the vector of observed effects (mean difference) and $\Gamma(\bullet)$ is a gamma function. Unfortunately, the complicated form of the full posterior (1) makes computation extremely difficult and one may have to resort to efficient simulation techniques such as the Markov Chain Monte Carlo (MCMC) for generating the posterior [16,17]. Via the Gibbs sampler algorithm [17], the technique works directly with the following conditional posterior distributions instead of the full posterior (1):

$$
\begin{aligned}
& \mathrm{p}\left[\phi_{\mathrm{i}} \mid \phi_{-\mathrm{i}}, \theta, \tau, \varphi, \mathrm{y}\right] \sqrt{\frac{\phi_{\mathrm{i}}}{2 \pi}} \exp \left[\frac{-\phi_{\mathrm{i}}}{2}\left(\mathrm{y}_{\mathrm{i}}-\varphi_{\mathrm{i}}\right)^{2}\right] \times \sqrt{\frac{\tau}{2 \pi}} \exp \left[\frac{-\tau}{2}\left(\varphi_{\mathrm{i}}-\theta\right)^{2}\right] \\
& \mathrm{p}[\theta \mid \phi, \tau, \varphi, \mathrm{y}] \prod_{\mathrm{i}=1}^{\mathrm{k}} \sqrt{\frac{\tau}{2 \pi}} \exp \left[\frac{-\tau}{2}\left(\varphi_{\mathrm{i}}-\theta\right)^{2}\right] \times \sqrt{\frac{v}{2 \pi}} \exp \left[\frac{-\mathrm{v}}{2}(\theta-\mu)^{2}\right]
\end{aligned}
$$





Objective Bayesian Random-Effect Meta-Analysis. Cardiolog Res Cardiovasc Med 1: 102. DOI: 10.29011/2575-7083.000002

$\mathrm{p}[\tau \mid \theta, \phi, \varphi, \mathrm{y}] \prod_{\mathrm{i}=1}^{\mathrm{k}} \sqrt{\frac{\tau}{2 \pi}} \exp \left[\frac{-\tau}{2}\left(\varphi_{\mathrm{i}}-\theta\right)^{2}\right] \times \frac{\eta^{\lambda}}{\Gamma(\lambda)} \tau^{\lambda-1} \exp [-\boldsymbol{\eta}]$

Where $\phi_{-i}$ represents the vector of all other trial-specific effects in studies other than $i$. The full posterior which contains the three quantities $\left(\theta, \phi_{\mathrm{i}} \tau\right.$, ) could be separated as three conditional posteriors with each featuring a quantity of concern explicitly. The advantage of performing computation on the conditional posteriors is that they have simpler structures. There is also no loss of information with the simplified forms as they are derived directly from the full posterior (1).

The rationale of Gibbs sampler is to cycle through the three conditional posteriors (2) for generating the estimates for the three desired quantities $\left(\theta, \phi_{\mathrm{i}} \tau\right)$ :

i. $\quad$ with initializing values $\varphi_{\mathrm{i}}^{(0)}=\mathrm{y}_{\mathrm{i}}, \theta^{(0)}=\sum_{\mathrm{i}=1}^{\mathrm{k}} \varphi_{\mathrm{i}} / \mathrm{k}$ and $\mathrm{t}_{i}^{(0)}=\mathrm{k} / \sum_{\mathrm{i}=1}^{\mathrm{n}}\left(\varphi_{\mathrm{i}}-\theta\right)^{2}$

ii. draw each $\phi_{i}$ randomly using its conditional posterior and the current values of $\theta$ and $\tau$

iii. draw $\theta$ randomly using its conditional posterior and the current values of $\phi$ and $\tau$

iv. draw $\tau$ randomly using its conditional posterior and the current values of $\phi$ and $\theta$

V. $\quad$ record the current values of $\phi, \theta$ and $\tau$

vi. repeat steps ii. to v. for a sufficiently large number of times, say 500 to 1,000

vii. the quantities generated represent a sample from the full posterior (1)

viii. summarize $\theta$ from the generated sample of posteriors by computing its mean, standard deviation and credible interval

By drawing a large number of values from these conditional posteriors (2), one is guaranteed that the full posterior (1) of the pooled effect could be obtained [18]. Since the above-mentioned Gibb sampling is iterative in nature, the generation of the estimated quantities depends on the initializing values. Under the suspicion that they are not representative of the full posterior (1), one should discard the beginning set of runs (burn-ins) before the updated posterior analysis is performed.

Upon completion the posterior distribution of the pooled effect $(\theta)$ could be summarized by means of a $95 \%$ credible interval. In conventional statistics, the random variables in a Confidence Interval (C.I.) are the limits and not the pooled effect $(\theta)$. With a $95 \%$ C.I. constructed, one may claim that the unknown pooled effect $(\theta)$ is contained in $95 \%$ of all possible intervals obtained with the same sampling method. In the case of Bayesian analysis, the C.I. provides a more natural and intuitive interpretation as $\theta$ may be stated to have a 0.95 probability of being within the interval.

A Stata 14.0 (Stata Corporation, Texas, USA.) programme was written for facilitating the Gibbs sampling. The programme allows users to read the data, enter the prior quantities and to specify the number of burn-ins and updates, with options for a graphical display of the iterative Gibbs sampling history. The graph provides a quick but reliable visual inspection on the convergence of the generated Markov chain.

The above formulation (1-2) illustrates the most important difference between the Bayesian and the conventional models. No prior distribution of $\theta$ is allowed in the conventional approach and the analysis is solely based on the likelihood functions. On the other hand, Bayesian analysis treats the unknown quantities (including the pooled effect $\theta$ ) as random variables and their priors have to be fixed. A typical question is raised with this difference in approach, that is, how Bayesian analysis cope if there are no prior knowledge of the quantities at all. The advice is to specify a non-informative distribution for the priors so that they have virtually no influence on the posterior. Not surprisingly, the posterior is then dominated by the likelihood and the result will be identical to the conventional analysis. However, the interpretation is philosophically different.

In the following analysis, the identified RCTs were first handled with the conventional random-effect model before they were reanalyzed with the proposed objective Bayesian model. A sensitivity analysis was then carried out with subjective priors in order to ascer- 


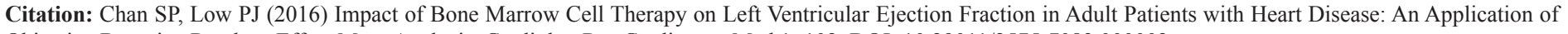
Objective Bayesian Random-Effect Meta-Analysis. Cardiolog Res Cardiovasc Med 1: 102. DOI: 10.29011/2575-7083.000002

tain the robustness of the objective Bayesian model. Statistical tests were conducted at $5 \%$ level of significance and with $95 \%$ C.I.s.

\section{Results}

The required data for meta-analysis are depicted in Table 1. Altogether, 54 RCTs involving 2,780 subjects (1,567 treated with BMCs and 1,213 served as controls) were included for the proposed meta-analysis. Detailed information on the search strategy, eligibility criteria, quality assessment and data abstraction are reported in references [2-8].

\begin{tabular}{|c|c|c|}
\hline Clinical Trial \& Year & $\begin{array}{c}\text { Mean Difference } \\
\dagger\end{array}$ & 95\% C.I. \\
\hline Ang 2008 & 1.46 & $-3.15-6.07$ \\
\hline Assmus 2006 (BMC)* & 4.10 & $2.18-6.02$ \\
\hline Assmus 2006 (CPC) & 0.80 & $-0.82-2.42$ \\
\hline Bartunek 2013* & 6.80 & $3.25-10.35$ \\
\hline Cao 2009* & 3.63 & $2.24-5.02$ \\
\hline Chen 2004* & 12.00 & $8.77-15.23$ \\
\hline Colombo 2011 & 3.80 & $-2.05-9.65$ \\
\hline Erbs 2005 & 7.20 & $-1.40-15.80$ \\
\hline Gao 2013 & 0.80 & $-1.14-2.74$ \\
\hline Ge 2006 & 6.70 & $-0.25-13.65$ \\
\hline Grajek 2010 & 3.07 & $-1.54-7.68$ \\
\hline Hendrikx 2006 & 2.50 & $-5.26-10.26$ \\
\hline Hirsch 2011 & -0.20 & $-2.44-2.04$ \\
\hline Huang 2006* & 2.90 & $1.27-4.53$ \\
\hline Huikuri $2008^{*}$ & 5.40 & $0.62-10.18$ \\
\hline Janssens 2006 & 1.20 & $-2.39-4.79$ \\
\hline Jazi 2012 & 4.00 & $-0.24-8.24$ \\
\hline Kang 2006 (AMI) & 5.20 & $-0.89-11.29$ \\
\hline Kang 2006 (OMI) & -0.20 & $-8.35-7.95$ \\
\hline Lipiec 2009 & -0.80 & $-4.80-3.20$ \\
\hline Lu 2013* & 5.40 & $1.30-9.50$ \\
\hline Lunde 2006 & 1.10 & $-2.99-5.19$ \\
\hline Maureira 2012 & 4.00 & $-3.63-11.63$ \\
\hline Meluzin 2006 & 2.00 & $-0.53-4.53$ \\
\hline Meyer 2006 & 2.80 & $-1.88-7.48$ \\
\hline
\end{tabular}

\begin{tabular}{|c|c|c|}
\hline Nogueira 2009 & 4.90 & $-4.48-14.28$ \\
\hline Penicka $2007^{*}$ & -5.10 & $-9.17--1.03$ \\
\hline Perin 2011 & -2.30 & $-7.67-3.07$ \\
\hline Perin 2012 (AHJ) & -2.00 & $-8.02-4.02$ \\
\hline Perin 2012 (JAMA)* & 2.70 & $0.36-5.04$ \\
\hline Piepoli 2013 & -3.00 & $-9.24-3.24$ \\
\hline Plewka 2011 & 5.30 & $-5.09-15.69$ \\
\hline Pokushalov 2010* & 6.10 & $5.04-7.16$ \\
\hline Quyyumi 2011 & 1.50 & $-4.42-7.42$ \\
\hline Ramshorst 2009* & 4.00 & $1.49-6.51$ \\
\hline Roncalli 2011 & -0.30 & $-2.90-2.30$ \\
\hline Ruan $2005^{*}$ & 9.17 & $0.77-17.57$ \\
\hline Schächinger $2006^{*}$ & 2.50 & $0.52-4.48$ \\
\hline Silva 2009 & 5.02 & $-4.99-15.03$ \\
\hline Srimahachota 2011 & -1.70 & $-7.41-4.01$ \\
\hline Suarez de Lezo $2007 *$ & 15.00 & $7.06-22.94$ \\
\hline Surder 2013 & 1.80 & $-1.12-4.72$ \\
\hline Traverse 2010 & -3.20 & $-10.32-3.92$ \\
\hline Traverse 2011 & -3.10 & $-7.28-1.08$ \\
\hline Traverse 2014 & 0.00 & $-3.02-3.02$ \\
\hline Tse 2007 & 4.10 & $-1.58-9.78$ \\
\hline Turan 2011* & 6.00 & $2.49-9.51$ \\
\hline Turan 2012* & 10.00 & $6.68-13.32$ \\
\hline Wohrle 2013 & -3.70 & $-9.44-2.04$ \\
\hline Yao 2008 & 0.80 & $-0.71-2.31$ \\
\hline Yao 2009* & 6.80 & $4.94-8.66$ \\
\hline Zhao 2008* & 9.35 & $5.45-13.25$ \\
\hline Li 2006* & 5.50 & $1.98-9.02$ \\
\hline \multicolumn{3}{|c|}{$\begin{array}{l}\dagger \text { Mean difference }>0 \text { : BMC was more effective in increasing LVE } \\
\text { than control } \\
* \text { Statistically significant }\end{array}$} \\
\hline
\end{tabular}

Table 1: Observed Mean Difference in LVEF (BMC versus Controls) in selected RCTs.

The majority of the studies showed that BMC might bring beneficial effects to patients suffering from AMI and IHD (mean 


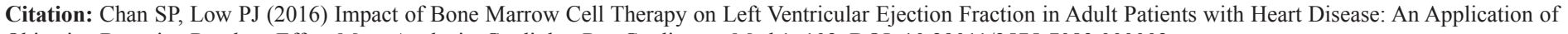
Objective Bayesian Random-Effect Meta-Analysis. Cardiolog Res Cardiovasc Med 1: 102. DOI: 10.29011/2575-7083.000002

difference $>0$, Table 1). However, only 19 selected trials reported significant results that favoured BMC.

The conventional random-effect model shows that $\mathrm{BMC}$ improved LVEF by about $+3.06 \%$ (95\% C.I.:2.11-4.01). The results are reported in Table 2 . Thus, there seems to be evidence suggesting that patients on BMC did enjoy modest improvement in LVEF when compared with their controls. The choice of a randomeffect model is justified with the test for heterogeneity $(\mathrm{p}<0.01)$. There was considerable heterogeneity as $\mathrm{I} 2=75.32 \%$. Moreover, there was also evidence of publication bias according to the funnel plot (Figure 1).

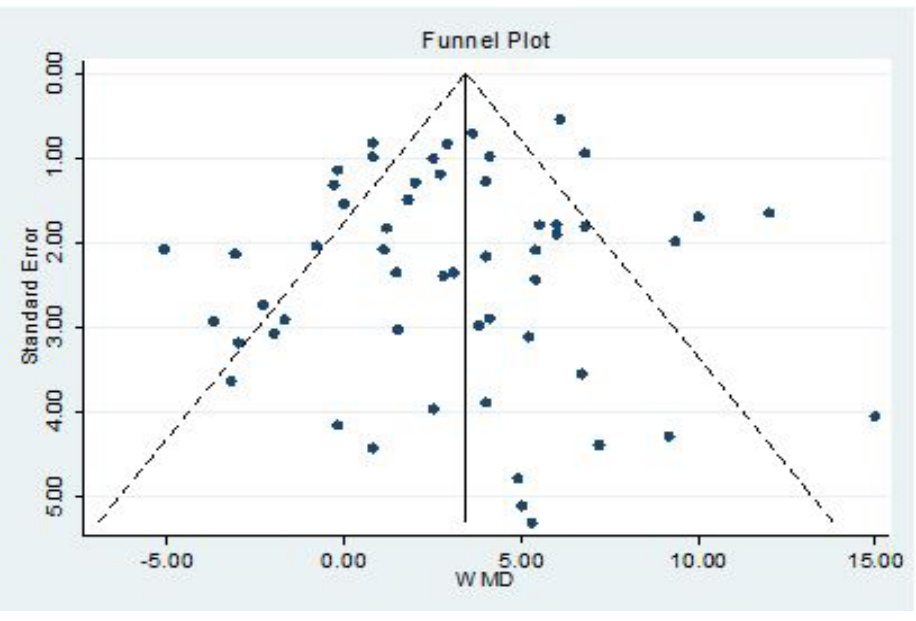

Figure 1: Funnel Plot.

The proposed Bayesian model was built next. The prior for $\theta$ was based on the conventional meta-analysis of 6 well-cited cohort studies published from 2002 to 2006 (Table 3) [3]. The pooled effect was $+3.83 \%$ with a precision of 0.55 . There was no strong evidence of heterogeneity ( $\mathrm{p}: 0.500$ ). While not widely regarded as confirmative as RCTs, cohort studies do provide useful evidence concerning treatment effects. As such, they are proposed as the source of evidence for constructing objective priors in this paper and meta-analysis involving RCTs at large. The prior for betweentrial precision $(\tau)$, however, was standardized with a gamma distribution $(\lambda=\eta=0.01)$. The choice of this distribution reflected the lack of prior evidence regarding between-trial precision. Also, the number of burn-ins was set a priori at 500 and the Markov chain would thereafter be run another 1,000 times before the final analysis was generated.

\begin{tabular}{|c|c|c|}
\hline & $\begin{array}{l}\text { Pooled Mean Dif- } \\
\text { ference in LVEF }\end{array}$ & $95 \% \mathrm{CI}^{*}$ \\
\hline $\begin{array}{c}\text { Conventional Random-Effect } \\
\text { Model }\end{array}$ & 3.06 & $2.11-4.01$ \\
\hline $\begin{array}{l}\text { Bayesian Random-Effect } \\
\text { Models: } \\
\text { abjective Prior (in } \\
\text { favour of BMC) } \\
\text { Prior Effect: }+3.83 \\
\text { Prior Precision of Effect: } 0.55\end{array}$ & 3.12 & $2.20-4.03$ \\
\hline $\begin{array}{l}\text { b) Neutral Prior (not in } \\
\text { favour of BMC or control) } \\
\text { Prior Effect: } 0.00 \\
\text { Prior Precision of Effect: } 0.55\end{array}$ & 2.60 & $1.67-3.54$ \\
\hline $\begin{array}{l}\text { Pessimistic Prior (in } \\
\text { favour of control) } \\
\text { Prior Effect: }-2.00 \\
\text { Prior Precision of Effect: } 0.55\end{array}$ & 2.32 & $1.35-3.29$ \\
\hline $\begin{array}{l}\text { Over-Optimistic Prior } \\
\text { (in favour of BMC) } \\
\text { Prior Effect: }+10.00 \\
\text { Prior Precision of Effect: } 0.55\end{array}$ & 3.98 & $2.99-4.97$ \\
\hline
\end{tabular}

Table 2: Results of Conventional and Bayesian Meta-Analyses.

\begin{tabular}{|c|c|c|}
\hline Cohort Study\& Year & Mean Difference & 95\% C.I. \\
\hline Bartunek 2005 & 2.80 & $-6.08-11.88$ \\
\hline Katritsis 2005 & 0.33 & $-5.57-6.23$ \\
\hline Mocini 2006 & 4.00 & $-1.29-9.29$ \\
\hline Perin 2004 * & 8.10 & $0.46-15.74$ \\
\hline Strauer 2002 & 1.00 & $-6.10-8.10$ \\
\hline Strauer 2005* & 7.00 & $1.07-12.93$ \\
\hline
\end{tabular}

Table 3: Observed Mean Difference in LVEF (BMC versus Control) in Cohort Studies.

The pooled effect of the objective Bayesian model turned out to be $+3.12 \%$ (Table 2 ). Since the $95 \%$ C.I. of $2.20-4.03$ does not contain zero, BMC is declared to be beneficial, although the effect was marginal. Thus, the objective Bayesian model confirmed the 


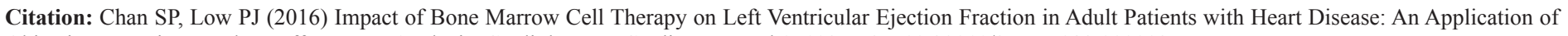
Objective Bayesian Random-Effect Meta-Analysis. Cardiolog Res Cardiovasc Med 1: 102. DOI: 10.29011/2575-7083.000002

superiority of BMC in improving LVEF. The normality assumption concerning the observed mean difference in LVEF was found to be reasonably valid (Figure 2). The objective Bayesian result was slightly more optimistic and precise than that of the conventional random-effect model in numerical sense, as the $95 \%$ C.I. of the pooled mean difference in LVEF was narrower. As depicted in Figure 3a, the Markov chain converged as the values fluctuated around $3.12 \%$.

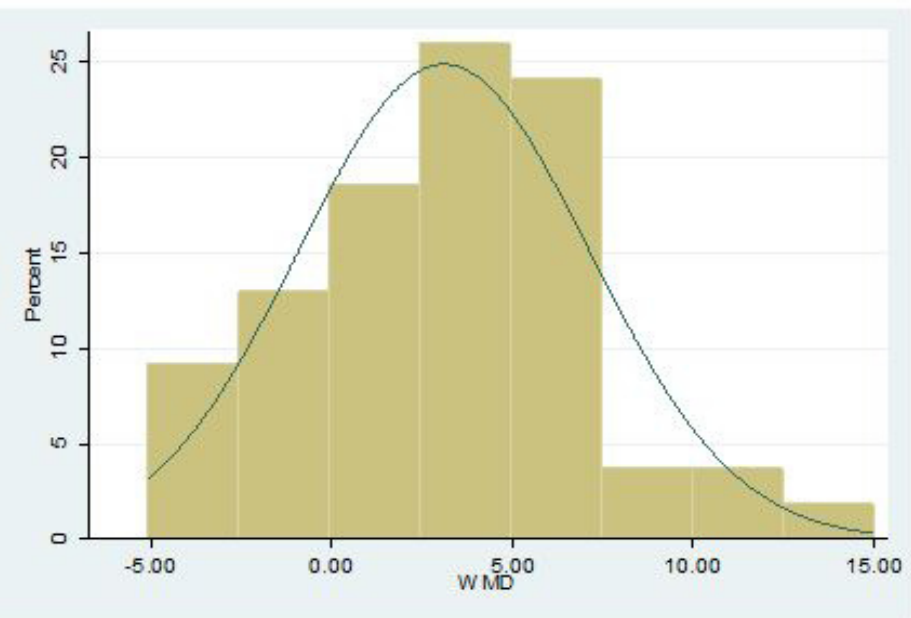

Figure 2: Histogram of Mean Difference in LVEF of selected RCTs.

It is helpful to consider various options of the Bayesian model and check if the results still hold under different circumstances. As the Bayesian model was based on the prior and likelihood one needs to know which component had a stronger influence on the posterior. The sensitivity analysis for ascertaining the influence of the prior on the posterior could also help to validate the robustness of the objective Bayesian model.

In the first attempt, a neutral subjective prior was applied. It was assumed that there was no difference in mean difference between BMC and control (i.e., $\theta=0$ ), while all other parameters remain unchanged. The pooled mean difference in LVEF was reduced to $+2.60 \%$ (95\% C.I.: 1.37-3.54; Table 2). In terms of magnitude, this was not a substantial reduction when compared with the previous analyses. It suggests that the prior had little impact on the posterior and the likelihood which contained all the RCT results dominated the analysis.

Next, a pessimistic prior was fitted (i.e., $\theta=-2.00$ ). One again the posterior was dominated by the likelihood with the pooled mean difference in LVEF estimated to be $+2.32 \%$ (95\% C.I.: $1.35-3.29$; Table 2 ). As readily seen the result was pretty much similar to that of the objective prior and the estimated BMC effect was modest.

Last but not least, an overwhelming optimistic prior (i.e., $\theta=+10.00$ ) also had little impact on the posterior result (Table 2). The pooled mean difference in LVEF went up marginally to 3.98
(95\% C.I.: 2.99-4.97; Table 3) owing to the pull effect of the overoptimistic prior. However, this was no significant change in the statistical sense as the $95 \%$ C.I.s overlap. In passing, note that the resultant posteriors based on the 4 different sets of priors ( 1 objective and 3 subjective) were normal and the Markov chains exhibited no obvious pattern of divergence after the burn-in values had been discarded (Figure $3 \mathrm{a}$ and $\mathrm{d}$ ).
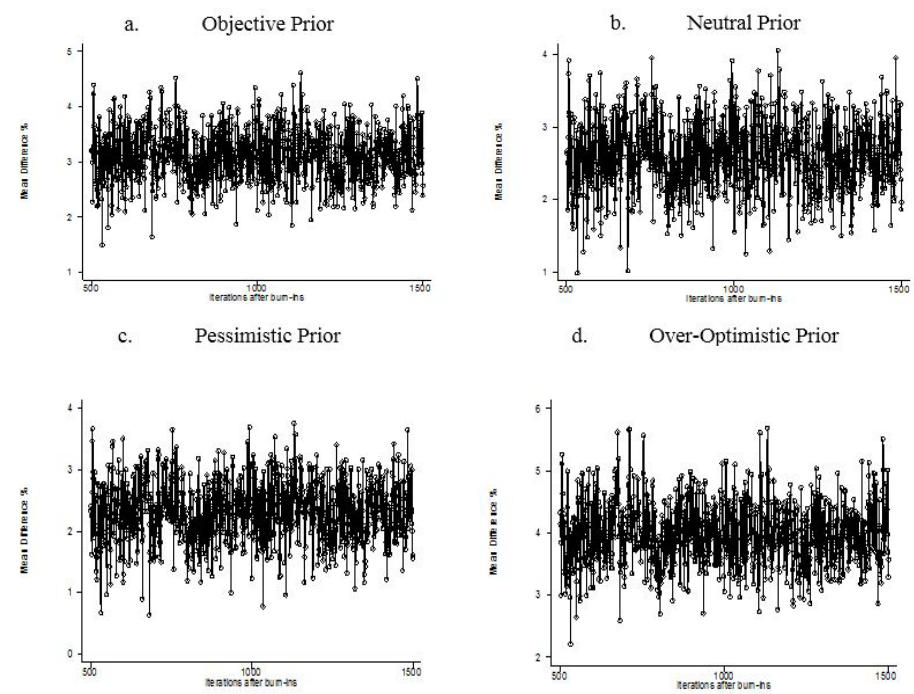

Figure 3(a-d): Iterative History of Bayesian Models after 500 Burn-Ins.

\section{Discussion and Conclusion}

The Bayesian model developed in this paper differs from the conventional approach in various aspects. First, it allows prior information-in the form of published evidence-to be incorporated into analysis. From the methodological point of view, it is costly to ignore such prior information if available. An objective Bayesian model could thus be built with priors based on published evidence. Unlike the conventional approach, Bayesian analysis is performed on the posterior distribution that summarizes both the prior- and data-based evidence, which the analysts have about the unknown pooled effect. Next, the Bayesian model is a randomeffect technique designed for situations where there is substantial heterogeneity among selected studies for meta-analysis, as it allows the observed effects (data) to vary around their individual studyspecific effects, which in turn belong to a distribution characterized by the pooled effect $(\theta)$. This makes sense given that there are inherent differences in the selected studies [14]. In addition, the model is hierarchical because more than one level of priors and likelihoods is specified. This sequence of priors and parameters constitute a model with an extended or hierarchical data structure.

By offering an alternative perspective in meta-analysis, the proposed Bayesian model provides more insights and relevant solutions to existing biomedical problems. In fact, one may view the 


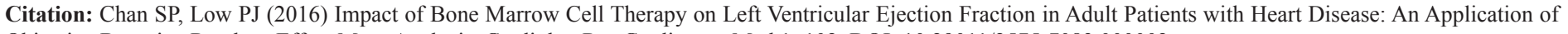
Objective Bayesian Random-Effect Meta-Analysis. Cardiolog Res Cardiovasc Med 1: 102. DOI: 10.29011/2575-7083.000002

conventional model as a special case within the broader framework of Bayesian methodology.

In future Bayesian meta-analysis the various endpoints may be analyzed jointly in a consolidated framework. For example, both the LVEF and the infarct size could be considered in a single Bayesian Structural Equation Model (SEM) which is one of the most popular areas of statistical research in recent years $[19,20]$. It is an advanced model constructed with the covariance matrix, thus allowing multiple correlated effects to be analyzed jointly in meaningful manner.

The above Bayesian meta-analyses show that the posteriors were not very much dominated by their respective priors. As such, the results generated by the proposed objective Bayesian model were reliable and credible. However, one needs to exercise care in citing the results as there was some evidence of publication bias, an issue not explicitly highlighted in references [2-8].

A more fundamental problem was surfaced in recent years. Despite making rapid progress in the field of cardiac cell therapy [1], there have been calls for improved quality in trials. While cardiac cell therapy has been reported to substantially increase cardiac function, the reported trials have differed in the effect sizes for some unknown reasons [19]. Moreover, there were over 600 discrepancies-reported facts that cannot be held valid owing to their logical incompatibility-discovered in 49 trials [19]. These include issues concerning the designs, methods and reported results. It was also pointed out that the number of discrepancies could be significantly associated with the reported effect size. This casts further doubts on the efficacy of BMC [19]. As such, one needs to exercise care when interpreting the results of individual RCTs and their meta-analyses despite a strong evidential support for BMC efficacy in statistical terms. This paper echoes the call for more large-scale RCTs to be conducted with a standard design before one could validate, verify and conclude the effectiveness of $\mathrm{BMC}$ as a reliable therapy option [2].

\section{References}

1. Wollert KC, Drexler H (2010) Cell therapy for the treatment of coronary heart disease: a critical approach. Nat Rev Cardiol 10: 204-215.

2. Hristov M, Heussen N, Schober A, Weber C (2006) Intracoronary infusion of autologous bone marrow cells and left ventricular function after acute myocardial infarction: a meta-analysis. J Cell Mol Med 10: 727-733.

3. Abdel-Latif A, Bolli R, Tleyjeh IM, Montori VM, Perin EC, et al. (2007) Adult bone-marrow derived cells for cardiac repair: A systematic review and meta-analysis. Arch Intern Med 167: 989-997.

4. Lipinski MJ, Biondi-Zoccai GG, Abbate A, Khianey R, Sheiban I, et al. (2007) Impact of intracoronary cell therapy on left ventricular function in the setting of acute myocardial infarction: a collaborative systematic review and meta-analysis of controlled clinical trials. J Am Coll Cardiol 50: $1761-1767$
5. Martin-Rendon E, Brunskill SJ, Hyde CJ, Stanworth SJ, Mathur A, et al. (2008) Autologous bone marrow stem cells to treat acute myocardial infarction: a systematic review. Eur Heart J 29: 1807-18.

6. Zhang SN, Sun AJ, Ge JB, Yao K, Huang ZY, et al. (2009) Intracoronary autologous bone marrow stem cells transfer for patients with acute myocardial infarction: a meta-analysis of randomised controlled trails. Int J Cardiol 136: 178-185.

7. Chan SP, Lee R, Poh KL (2009) A Bayesian review of the meta-analysis on the efficacy of bone marrow-derived cells transplantation in adult patients with heart disease. Proc World Congress on Engineering 1287-1291.

8. Afzal MR, Samanta A, Shah ZI, Jeevanantham V, Abdel-Latif A, et al. (2015) Adult bone marrow cell therapy for ischemic heart disease: evidence and insights from randomized controlled trials. Circ Res 117: $558-575$.

9. Goodman SN (2009) Building a Bayesian bridge from evidence to guidelines. Arch Intern Med 169: 1436-1437.

10. Goodman SN (2013) Bayesian methods for evidence evaluation: are we there yet?. Circulation 127: 2367-2369.

11. Fleiss JL (1993) The statistical basis of meta-analysis. Stat Methods Med Res 2: 121-145.

12. Piranfar MA, Karvandi M, Yazdani S, Pishgahi M, Mehdizadeh M, et al. (2012) Bone marrow transplantation may augment cardiac systolic function in patients with a reduced left ventricular election fraction. $\mathrm{J}$ Cardiovasc Dis Res 3: 310-314.

13. Gelman A (2008) Objections to Bayesian statistics. Bayesian Analysis 3: $445-450$.

14. Jeevanantham V, Afzal MR, Zuba-Surma EK, Dawn B (2013) Clinical trials of cardiac repair with adult bone marrow-derived cells. Methods Mol Biol 1036: 179-205.

15. Bayes T (2002) An essay towards solving a problem in the doctrine of chance. In: Swinburne R, (ed.) Bayes's Theorem. Oxford: Oxford University Press, USA.

16. Carlin BP, Gelman A, Neal RM (1998) Markov chain Monte Carlo in practice: a roundtable discussion. J Am Stat Assoc 52: 93-100.

17. Casella G, George El (1992) An introduction to Gibbs sampling. Ann Math Stat 46: 167-174.

18. Zellner A, Min CK (1995) Gibbs sampler: convergence criteria. J Am Stat Assoc 90: 921-927.

19. Nowbar AN, Mielewczik M, Karavassilis M, Debhi HM, Shun-Shin MJ, et al. (2014) Discrepancies in autologous bone marrow stem cell trials and enhancement of ejection fraction (DAMASCENE): weighted regression and meta-analysis. BMJ 348: 1-9.

20. Muthén B, Asparouhov T (2012) Bayesian SEM: A more flexible representation of substantive theory. Psychological Methods 17: 313-315. 